\title{
ON COHERENT SHEAVES OF SMALL LENGTH ON THE AFFINE PLANE
}

\author{
RICCARDO MOSCHETTI AND ANDREA T. RICOLFI
}

\begin{abstract}
We classify coherent modules on $k[x, y]$ of length at most 4 and supported at the origin. We compare our calculation with the motivic class of the moduli stack parametrizing such modules, extracted from the Feit-Fine formula. We observe that the natural torus action on this stack has finitely many fixed points, corresponding to connected skew Ferrers diagrams.
\end{abstract}

\section{CONTENTS}

1. Introduction

2. Main strategy and conventions 2

3. Motivic interpretation 5

4. Modules of length three

5. Modules of length four 8

6. Torus action 14

References 16

\section{INTRODUCTION}

Finitely generated modules over principal ideal domains satisfy a known structure theorem, and finitely generated modules over a Dedekind domain were characterized by Steinitz over a century ago [Ste11]. In this paper we study modules of finite length over the polynomial ring

$$
A=k[x, y],
$$

where $k$ is an algebraically closed field of characteristic zero. These naturally correspond to coherent sheaves supported on finitely many points of the affine plane $\mathbb{A}^{2}$. We only consider modules entirely supported at the origin, as every other module is isomorphic to a direct sum of modules of this type.

Among all such modules are artinian ring quotients of $A$, corresponding to points of the punctual Hilbert scheme

$$
\operatorname{Hilb}^{n}\left(\mathbb{A}^{2}\right)_{0} \subset \operatorname{Hilb}^{n}\left(\mathbb{A}^{2}\right) .
$$

It is known that there are infinitely many isomorphism types of artinian $k$-algebras of length 8. Those of length at most 6 have been completely described by Briançon [Bri77]. Moreover, Poonen has classified isomorphism types of $k$-algebras of dimension up to 6 and proves there are infinitely many types in dimension $n \geq 7$ [Poo08].

The problem of classifying $A$-modules of finite length up to $A$-linear isomorphism, that we study here, is equivalent to the one of classifying pairs of commuting linear

E-mail addresses: riccardo.moschetti@uis.no, andrea.ricolfi@uis.no.

2010 Mathematics Subject Classification. 14D23,14C05.

Key words and phrases. Coherent sheaves, Grothendieck ring of stacks. 
transformations on a finite dimensional vector space; the latter is known to contain the problem of classifying arbitrary tuples of commuting linear transformations, by work of Gelfand and Ponomarev [GP69].

We could not find a reference in the literature for the classification of isomorphism classes of $A$-modules of length $n>2$. We deal with length $n=3$ and $n=4$ in this work.

1.1. Main result. For simplicity, we state our classification in terms of (isomorphism classes of) indecomposable modules only, but see Tables 1 and 2 for complete lists including the decomposable ones. All modules are supported entirely on $\mathfrak{m}=(x, y)$, the ideal of the origin. Our main result is the following.

THEOREM 1. The indecomposable modules oflength 3 are either structure sheaves or the distinguished module $\operatorname{Hom}_{k}\left(A / \mathfrak{m}^{2}, k\right)$. In the length 4 case, besides structure sheaves, there are two families $\mathcal{F}_{1}$ and $\mathcal{F}_{2}$ of indecomposable modules, both isomorphic to $\mathbb{P}^{1}$.

The stack of $A$-modules of length $n$ will be denoted $\mathcal{C}(n)$ throughout. We let

$$
\mathcal{C}(n)_{0} \subset \mathcal{C}(n)
$$

be the closed substack parametrizing modules entirely supported at the origin. Even though these stacks are not well understood in general, remarkably their motivic classes in the Grothendieck ring of stacks can be computed for arbitrary $n$, by means of the Feit-Fine formula [FF60, BBS13, BM15]. The knowledge of the motivic aspect of the theory is for us both a motivation to tackle the classification problem, and a way to check our results. More precisely, our strategy goes as follows. We stratify $\mathcal{C}(n)_{0}$ by locally closed substacks

$$
\mathcal{X}_{r}(n) \subset \mathcal{C}(n)_{0},
$$

each parametrizing modules $M$ such that $\operatorname{dim}_{k} M / \mathfrak{m} \cdot M=r$. In other words, we study modules $M$ by means of their discrete invariant

$$
r_{M}=\text { minimal number of generators of } M .
$$

We then analyze each stratum separately, and we compute its motivic class. Since we are inside a quotient stack, this requires us to compute all possible automorphism groups. To confirm our calculation we verify that the sum

$$
\sum_{r=1}^{n}\left[\mathcal{X}_{r}(n)\right] \in K_{0}\left(\mathrm{St}_{k}\right)
$$

reconstructs the class $\left[\mathcal{C}(n)_{0}\right]$ predicted by the Feit-Fine formula.

In Section 6 we study the natural action of the torus $\mathbf{T}=\mathbb{G}_{m}^{2}$ on the moduli stack $\mathcal{C}(n)$ and we prove that it has finitely many fixed points, corresponding to certain types of skew Ferrers diagrams. We will also observe that the generating function for the numbers of indecomposable torus-fixed modules has a well-known combinatorial interpretation in terms of parallelogram polyominoes.

\section{MAIN STRATEGY AND CONVENTIONS}

Let $k$ be an algebraically closed field of characteristic zero. We let

$$
\mathfrak{m} \subset A=k[x, y]
$$


be the maximal ideal of the origin in $\mathbb{A}^{2}$. All $A$-modules $M$ are assumed to be of finite length and entirely supported at $\mathrm{m}$. Note that since the function

$$
\mathrm{r}: M \mapsto \operatorname{dim}_{k} M / \mathfrak{m} \cdot M
$$

is upper semi-continuous, there are well-defined locally closed substacks

$$
\mathcal{X}_{r}(n) \subset \mathcal{C}(n)_{0}
$$

parametrizing modules with $r$ as minimal number of generators. The motivic class of $\mathcal{X}_{r}(n)$ makes sense, and we have a decomposition

$$
\left[\mathcal{C}(n)_{0}\right]=\sum_{r=1}^{n}\left[\mathcal{X}_{r}(n)\right] \in K_{0}\left(\mathrm{St}_{k}\right)
$$

See [Eke09] for an introduction to the Grothendieck group of algebraic stacks.

If $M$ lies in $\mathcal{X}_{r}(n)$, we will find useful to fix a $k$-linear basis

$$
\left\{v_{1}, v_{2}, \ldots, v_{n}\right\} \subset M
$$

such that the first $r$ vectors generate the module, and $v_{r+1}, \ldots, v_{n}$ generate the submodule

$$
\mathfrak{m} \cdot M \subset M
$$

as a $k$-vector space.

LEMMA 2.1. With the above choice of basis, $x \cdot v_{i}$ and $y \cdot v_{i}$ belong to $\mathfrak{m} \cdot M$.

PRoOF. Let $\pi: M \rightarrow M / \mathfrak{m} \cdot M$ be the canonical projection. By our choice of basis, $v_{r+1}, \ldots, v_{n}$ form a $k$-basis of $\mathfrak{m} \cdot M$ and $M / \mathfrak{m} \cdot M$ is generated over $k$ by the images of $v_{1}, \ldots, v_{r}$. Writing $x \cdot v_{i}=\sum_{j=1}^{n} a_{j} v_{j}$ for some $a_{j} \in k$, from the relation

$$
0=x \cdot \pi\left(v_{i}\right)=\pi\left(x \cdot v_{i}\right)=\sum_{j=1}^{n} a_{j} \pi\left(v_{j}\right)=\sum_{j=1}^{r} a_{j} \pi\left(v_{j}\right)
$$

one deduces that $a_{j}=0$ for $j=1, \ldots, r$. Therefore $x \cdot v_{i}=\sum_{j=r+1}^{n} a_{j} v_{j}$ belongs to $\mathfrak{m} \cdot M$, and similarly for $y \cdot v_{i}$.

The lemma says that after fixing a suitable basis for $M$ one can see multiplication by $x$ and $y$ as $k$-linear maps

$$
\left\langle v_{1}, \ldots, v_{r}\right\rangle_{k} \underset{y}{\stackrel{x}{\longrightarrow}} \mathfrak{m} \cdot M .
$$

If the additional condition $x \cdot\left(y \cdot v_{i}\right)=y \cdot\left(x \cdot v_{i}\right)$ is fulfilled for $i=1, \ldots, r$ then the two $k$-linear maps above characterize $M$. Such point of view will be essential when dealing with length 4 modules satisfying $r_{M}=2$. Then our strategy will be to classify all pairs of linear maps as above, for each choice of length two submodule $\mathfrak{m} \cdot M \subset M$.

We anticipate here that we will sometimes describe modules by means of their "multiplication table". This is just a way to represent the action of $x$ and $y$ on a chosen basis $v_{1}, \ldots, v_{n}$. The first $r$ entries of the table are to be filled in according to Lemma 2.1, whereas the last $n-r$ are describing the submodule $\mathfrak{m} \cdot M \subset M$. Occasionally, we will encounter modules that can be visually represented as certain types of skew Ferrers diagrams, see Example 2.2 below. In Section 6 we will see that these special modules are the finitely many fixed points of the natural torus action on the moduli stack $\mathcal{C}(n)$. For instance, as is well-known, classical Ferrers diagrams correspond to the fixed points of the torus action on $\operatorname{Hilb}^{n}\left(\mathbb{A}^{2}\right)$, studied in [ES87]. Recall that Ferrers diagrams (also called Young diagrams) correspond to ordinary partitions of integers, whereas a skew Ferrers diagram is a difference of two Ferrers diagrams. 
Our convention for (skew) Ferrers diagrams is to use the French notation; when a module can be represented by a skew Ferrers diagram, we understand multiplication by $x$ (resp. $y$ ) in the module as shifting position to the right (resp. to the top) in the diagram. The following example illustrates our conventions.

Example 2.2. Consider the module $M=A / \mathfrak{m}^{2}=k[x, y] /\left(x^{2}, x y, y^{2}\right)$. This is the unique non-curvilinear structure sheaf of length 3 , with natural $k$-basis $1, x, y$. The multiplication tables

\begin{tabular}{llll} 
& 1 & $x$ & $y$ \\
\hline$x \cdot$ & $x$ & 0 & 0 \\
$y \cdot$ & $y$ & 0 & 0 \\
\hline
\end{tabular}

\begin{tabular}{cccc} 
& $1^{*}$ & $x^{*}$ & $y^{*}$ \\
\hline$x \cdot$ & 0 & $1^{*}$ & 0 \\
$y \cdot$ & 0 & 0 & $1^{*}$ \\
\hline
\end{tabular}

describe, respectively, the $A$-linear structure of $M$ and of its $k$-linear dual $M^{*}=$ $\operatorname{Hom}_{k}(M, k)$. These tables can be represented as diagrams
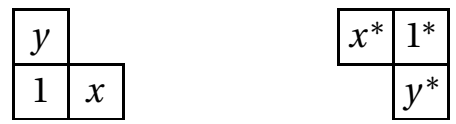

where the first one is a classical Ferrers diagram and the second one is the skew Ferrers diagram corresponding to $M^{*}$. We will see as a part of Theorem 1 that $M^{*}$ is the unique indecomposable module of length 3 that is not a structure sheaf. In the following we will avoid writing the name of the generators inside the diagrams.

As a warm-up to illustrate our classification technique, we now describe all isomorphism types of $A$-modules belonging to the stratum $\mathcal{X}_{n-1}(n) \subset \mathcal{C}(n)_{0}$. Recall that by $r=r_{M}$ we mean the minimal number of generators of $M$.

Proposition 2.3. Any module $M$ of length $n \geq 3$ with $r_{M}=n-1$ is isomorphic to $k^{n-3} \oplus N$, where $N$ has length 3 and $r_{N}=2$.

Proof. Any $A$-module $N$ of length 3 with $r_{N}=2$ gives rise to $M=k^{n-3} \oplus N$, with $r_{M}=n-1$. Conversely, if $M$ is generated over $A$ by $v_{1}, \ldots, v_{n-1}$ and $v_{n}$ generates $\mathfrak{m} \cdot M=k$, then the multiplication table for $M$ looks like

\begin{tabular}{cccccc} 
& $v_{1}$ & $v_{2}$ & $\cdots$ & $v_{n-1}$ & $v_{n}$ \\
\hline$x \cdot$ & $a_{1} v_{n}$ & $a_{2} v_{n}$ & $\cdots$ & $a_{n-1} v_{n}$ & 0 \\
$y \cdot$ & $b_{1} v_{n}$ & $b_{2} v_{n}$ & $\cdots$ & $b_{n-1} v_{n}$ & 0 \\
\hline
\end{tabular}

where $a_{i}$ and $b_{i}$ are scalars in $k$. Up to relabeling the generators we can assume either $a_{1}$ or $b_{1}$ to be nonzero. We deal with the former case, since the latter is completely symmetric.

If $a_{1} \neq 0$ we can assume it is equal to 1 . Replacing $v_{i}$ by $v_{i}-a_{i} v_{1}$ for $i=2, \ldots, n-1$ we get the multiplication table

\begin{tabular}{cccccc} 
& $v_{1}$ & $v_{2}$ & $\cdots$ & $v_{n-1}$ & $v_{n}$ \\
\hline$x \cdot$ & $v_{n}$ & 0 & $\cdots$ & 0 & 0 \\
$y \cdot$ & $b_{1} v_{n}$ & $b_{2}^{\prime} v_{n}$ & $\cdots$ & $b_{n-1}^{\prime} v_{n}$ & 0 \\
\hline
\end{tabular}

If $b_{2}^{\prime}=0$ then $M=\left\langle v_{2}\right\rangle_{k} \oplus F$ for $F$ a module of length $n-1$, and the result follows by induction. If $b_{2}^{\prime} \neq 0$, we can assume $b_{2}^{\prime}=1$ and replace $v_{i}$ by $v_{i}-b_{i}^{\prime} v_{2}$ for $i=3, \ldots, n-1$. 
This yields

\begin{tabular}{ccccccc} 
& $v_{1}$ & $v_{2}$ & $v_{3}$ & $\cdots$ & $v_{n-1}$ & $v_{n}$ \\
\hline$x \cdot$ & $v_{n}$ & 0 & 0 & $\cdots$ & 0 & 0 \\
$y \cdot$ & $b_{1} v_{n}$ & $v_{n}$ & 0 & $\cdots$ & 0 & 0 \\
\hline
\end{tabular}

so that $M=k^{n-3} \oplus F$ where $F$ is a length 3 module generated by $v_{1}$ and $v_{2}$.

\section{MOTIVIC INTERPRETATION}

The Grothendieck ring of algebraic stacks $K_{0}\left(\mathrm{St}_{k}\right)$ carries a power structure naturally extending the one present on the classical Grothendieck ring of varieties, which is due to Gusein-Zade, Luengo and Melle-Hernández [GLM04]. We refer to [BM15] for more details. Define the generating functions

$$
\mathrm{C}(t)=\sum_{n=0}^{\infty}[\mathcal{C}(n)] t^{n}, \quad \mathrm{C}_{0}(t)=\sum_{n=0}^{\infty}\left[\mathcal{C}(n)_{0}\right] t^{n} .
$$

As observed in [BM15], these power series are related, via the power structure on $K_{0}\left(\mathrm{St}_{k}\right)$, by $\mathrm{C}(t)=\mathrm{C}_{0}(t)^{\mathbb{L}^{2}}$. We now recall a formula for $\mathrm{C}(t)$ originally proved by Feit and Fine $\left[\right.$ FF60] in the context of point counting over $\mathbb{F}_{q}$.

THEOREM 3.1. [BBS13, BM15] In $K_{0}\left(\mathrm{St}_{\mathbb{C}}\right) \llbracket t \rrbracket$, one has the formula

$$
\mathrm{C}(t)=\prod_{k=1}^{\infty} \prod_{m=1}^{\infty}\left(1-\mathbb{L}^{2-k} t^{m}\right)^{-1} .
$$

Remark 3.2. The above relation, proved over $\mathbb{C}$ in the references given, holds in the Grothendieck ring of stacks over any algebraically closed field $k$. The main technical result needed for the proof in [BM15], besides the existence of the Jordan normal form, is that $K_{0}\left(\mathrm{St}_{k}\right)$ is isomorphic to the localization of $K_{0}\left(\operatorname{Var}_{k}\right)$ at the classes $\mathbb{L}$ and $\mathbb{L}^{n}-1$ for $n>0$. This is true over any field by [Eke09, Theorem 1.2].

The properties of the power structure [BM15] allow one to deduce

$$
C_{0}(t)=C(t)^{\mathbb{L}^{-2}}=\prod_{k=1}^{\infty} \prod_{m=1}^{\infty}\left(1-\mathbb{L}^{-k} t^{m}\right)^{-1} .
$$

Expanding the above series, one finds

$$
\mathrm{C}_{0}(t)=1+\frac{1}{\mathbb{L}-1} t+\left(\frac{1}{\left[\mathrm{GL}_{2}\right]}+\frac{\mathbb{L}+1}{\mathbb{L}(\mathbb{L}-1)}\right) t^{2}+\cdots
$$

The geometric interpretation of the first coefficients is clear:

(0) the motivic class of $\mathcal{C}(0)_{0}=\operatorname{Spec} k$ is just 1 ;

(1) $1 /(\mathbb{L}-1)$ is the motivic class of the stack $\mathcal{C}(1)_{0}=B \mathbb{G}_{m}$, which has only one point (corresponding to the module $k=A / \mathfrak{m}$ ), weighted by its automorphism group $\mathbb{G}_{m}$;

(2) the stack $\mathcal{C}(2)_{0}$ decomposes as $\mathcal{X}_{2}(2)=\mathrm{BGL}_{2}$ (corresponding to $k \oplus k$ ) union $\mathcal{X}_{1}(2)=\mathbb{P}^{1} / \mathbb{G}_{a} \rtimes \mathbb{G}_{m}$, where the projective line $\mathbb{P}^{1}=\mathbb{P}\left(\mathfrak{m} / \mathfrak{m}^{2}\right)$ represents the punctual Hilbert scheme $\operatorname{Hilb}^{2}\left(\mathbb{A}^{2}\right)_{0}$, which parametrizes structure sheaves of length 2 supported at the origin (each having automorphism group $\mathbb{G}_{a} \rtimes$ $\left.\mathbb{G}_{m}\right)$. 
We aim at giving a similar interpretation of the next two coefficients of $C_{0}(t)$, using our stratification.

When we present a (substack of a) stratum $\mathcal{X}_{r}(n)$ as a quotient stack $Y / G$, we will say that any module belonging to this stratum has motivic contribution the motive of the scheme $Y$.

3.1. Automorphism groups. The following general result on automorphism groups of quiver representations is going to help us compute all automorphism groups of $A$-modules of finite length.

THEOREM 3.3. [Bri12, Prop. 2.2.1] Let $M$ be a finite dimensional representation of a quiver $Q$. Then $\operatorname{Aut}(M)$ is a connected linear algebraic group, with a decomposition

$$
\operatorname{Aut}(M)=U \rtimes \prod_{i=1}^{s} \mathrm{GL}_{m_{i}},
$$

where $U$ is a closed normal unipotent subgroup and $m_{1}, \ldots, m_{s}$ are the multiplicities of the indecomposable summands of $M$.

We apply this result to the quiver $Q$ consisting of one node and two loops. The category $\operatorname{Rep}(Q)$ of representations of $Q$ is equivalent to the category of left modules over the path algebra of $Q$, which is the non-commutative algebra $k Q=k\langle x, y\rangle$. We need to consider the quiver with relations $(Q, I)$, where $I \subset k Q$ is the two-sided ideal generated by the single commutator $x y-y x$. Then the category of representations of $(Q, I)$ is a full subcategory of $\operatorname{Rep}(Q)$, naturally equivalent to the category of modules over $k Q / I=k[x, y]=A$.

Theorem 3.3 implies in particular that, if $M$ is an $A$-module of finite length, its automorphism group is a special algebraic group. Indeed, the GL factors are themselves special, every unipotent group in characteristic zero is an iterated extensions of copies of $\mathbb{G}_{a}$ (which is special), and any (semidirect) product of special groups is special. This fact is crucial for us: the Grothendieck ring $K_{0}\left(\mathrm{St}_{k}\right)$ can be characterized as the localization of $K_{0}\left(\operatorname{Var}_{k}\right)$ at the classes of special algebraic groups, and the upshot is that when a variety $Y$ is acted on by a special group $G$, the motivic class of the quotient stack $\mathscr{Y}=Y / G$ can be computed as

$$
[\mathscr{Y}]=[Y] /[G] \in K_{0}\left(\mathrm{St}_{k}\right) .
$$

In Tables 1 and 2, the column indicating the motive of the automorphism groups is obtained directly from Theorem 3.3. Note that in order to compute this class, one only needs the indecomposable factors of the module, and the dimension of the automorphism group $\operatorname{Aut}_{A}(M)$. The latter is an elementary calculation and easily gives the number of copies of $\mathbb{G}_{a}$ appearing in the unipotent factor $U$.

As an example, consider the stack of coherent sheaves of length $n$ on affine space $\mathbb{A}^{d}$, supported at the origin. By Theorem 3.3 , its locally closed substack parametrizing structure sheaves has motivic class

$$
\frac{\left[\operatorname{Hilb}^{n}\left(\mathbb{A}^{d}\right)_{0}\right]}{\mathbb{L}^{n-1}(\mathbb{L}-1)} .
$$

Indeed, $\mathscr{O}_{Z}$ is indecomposable and $\operatorname{Aut}\left(\mathscr{O}_{Z}\right)$ has dimension $n$ for all fat points $Z \subset \mathbb{A}^{d}$, since an automorphism is determined by the image of $1 \in \mathscr{O}_{Z}$. If $d=2$, this computes the class

$$
\left[\mathcal{X}_{1}(n)\right]=\frac{\left[\operatorname{Hilb}^{n}\left(\mathbb{A}^{2}\right)_{0}\right]}{\mathbb{L}^{n-1}(\mathbb{L}-1)} .
$$


To obtain the motive of the punctual Hilbert scheme, one can use the expansion

$$
\sum_{n=0}^{\infty}\left[\operatorname{Hilb}^{n}\left(\mathbb{A}^{2}\right)_{0}\right] t^{n}=\prod_{m \geq 1}\left(1-\mathbb{L}^{m-1} t^{m}\right)^{-1}
$$

This follows directly from the motivic version of Göttsche's formula [Göt01], as it is also explained in [GLM04].

Example 3.4. The curvilinear locus inside the punctual Hilbert scheme is known to be a dense open subset, fibred over $\mathbb{P}^{1}$ with fibre $\mathbb{A}^{n-2}$ [Bri77]. Hence the motivic class $\left[\operatorname{Hilb}^{n}\left(\mathbb{A}^{2}\right)_{0}\right]$ always decomposes as $\mathbb{L}^{n-2}(\mathbb{L}+1)$ plus the class of the noncurvilinear locus. If $n=3$, the latter is just a single point corresponding to $A / \mathfrak{m}^{2}$, hence we get

$$
\left[\operatorname{Hilb}^{3}\left(\mathbb{A}^{2}\right)_{0}\right]=\mathbb{L}(\mathbb{L}+1)+1
$$

For higher $n$, one has to expand (3.3) in order to extract the motive of the punctual Hilbert scheme.

\section{Modules of Length THREE}

We have three strata $\mathcal{X}_{r}(3) \subset \mathcal{C}(3)_{0}$. Structure sheaves correspond to $r=1$ and contribute

$$
\left[\mathcal{X}_{1}(3)\right]=\frac{\mathbb{L}(\mathbb{L}+1)+1}{\mathbb{L}^{2}(\mathbb{L}-1)}
$$

by formula (3.2). We have $\mathcal{X}_{3}(3)=\mathrm{BGL}_{3}$, and we compute $\mathcal{X}_{2}(3)$ in the next proposition.

Proposition 4.1. The modules of length 3 having $r=2$ are $\left(A / \mathrm{m}^{2}\right)^{*}$ and those of the form $k \oplus \mathscr{O}_{Z}$, where $Z \subset \mathbb{A}^{2}$ is a subscheme of length 2 . The latter form a family isomorphic to $\mathbb{P}^{1}$.

PROOF. This can be extracted from the proof of Proposition 2.3. Indeed, the decomposable modules (necessarily of the form $k \oplus \mathscr{O}_{Z}$ ) occur when $b_{2}^{\prime}=0$, and the only new module corresponds to the final table, which represents (independently upon the choice of $\left.b_{1}\right)$ the indecomposable module $\left(A / \mathfrak{m}^{2}\right)^{*}$.

This proves the part of Theorem 1 concerning $n=3$. The skew Ferrers diagrams representing a length 3 module are precisely

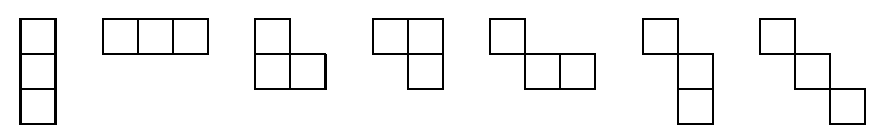

where the first 4 are indecomposable. The automorphism groups of all modules are computed through Theorem 3.3. See Table 1 for the complete list.

Remark 4.2. The coefficient of $t^{3}$ in (3.1) can be computed to be

$$
\left[\mathcal{C}(3)_{0}\right]=\frac{1}{\left[\mathrm{GL}_{3}\right]}\left(\mathbb{L}^{8}+\mathbb{L}^{7}+\mathbb{L}^{6}-\mathbb{L}^{5}-\mathbb{L}^{4}\right)
$$

The sum of the motives in the rightmost column of Table 1, each divided by the motive of the corresponding automorphism group, recovers precisely this class, confirming our calculation. 


\begin{tabular}{cccc}
$r$ & $\mathcal{C}(3)_{0}$ & {$\left[\operatorname{Aut}_{A}(M)\right]$} & Motivic contribution \\
\hline 1 & $\mathscr{O}_{Z}$ & $\mathbb{L}^{2}(\mathbb{L}-1)$ & $\mathbb{L}(\mathbb{L}+1)+1$ \\
2 & $\left(A / \mathfrak{m}^{2}\right)^{*}$ & $\mathbb{L}^{2}(\mathbb{L}-1)$ & 1 \\
2 & $k \oplus \mathscr{O}_{Z}$ & $\mathbb{L}^{3}(\mathbb{L}-1)^{2}$ & $\mathbb{L}+1$ \\
3 & $k^{\oplus 3}$ & {$\left[\mathrm{GL}_{3}\right]$} & 1 \\
\hline
\end{tabular}

TABLE 1 . All $k[x, y]$-modules of length 3 supported at the origin, along with the class of their automorphism group and their motivic contribution. The first two rows describe indecomposable modules.

\section{MOdUles OF LENGTH FOUR}

We need to analyze the strata $\mathcal{X}_{r}(4) \subset \mathcal{C}(4)_{0}$ for $r=1,2$, 3, 4. Expanding (3.3) we find

$$
\left[\operatorname{Hilb}^{4}\left(\mathbb{A}^{2}\right)_{0}\right]=\mathbb{L}^{3}+2 \mathbb{L}^{2}+\mathbb{L}+1,
$$

and this determines the class of $\mathcal{X}_{1}(4)$ through (3.2). On the other hand, $\mathcal{X}_{4}(4)=$ $\mathrm{BGL}_{4}$, and combining Propositions 2.3 and 4.1 with one another completely describes the stratum $\mathcal{X}_{3}(4)$ as follows.

Proposition 5.1. Let $M$ be a module of length 4 with $r_{M}=3$. Then either $M \cong$ $k \oplus\left(A / \mathfrak{m}^{2}\right)^{*}$, or $M \cong k^{2} \oplus \mathscr{O}_{Z}$, where $\mathscr{O}_{Z}$ is a structure sheaf of length 2 .

The three skew Ferrers diagrams determined by Proposition 5.1 are depicted in Figure 1 below.
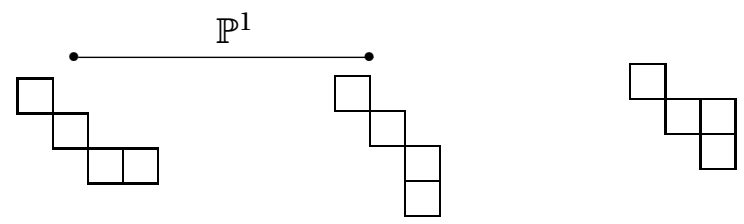

Figure 1. The decomposable modules of Proposition 5.1. The $\mathbb{P}^{1}$ represents the family $k^{2} \oplus \mathscr{O}_{Z}$, whereas the isolated diagram represents $k \oplus\left(A / \mathfrak{m}^{2}\right)^{*}$.

It remains to identify the stratum

$$
\mathcal{X}_{2}(4) \subset \mathcal{C}(4)_{0}
$$

corresponding to modules with $\operatorname{dim}_{k} M / \mathfrak{m} \cdot M=2$. These come in two types: either

(a) $\mathfrak{m} \cdot M=\mathscr{O}_{Z}$, a structure sheaf of length 2 , or

(b) $\mathfrak{m} \cdot M=k \oplus k$.

The families $\mathcal{F}_{1}$ and $\mathcal{F}_{2}$ of indecomposable modules mentioned in Theorem 1 will arise from case (a) and (b), treated in Proposition 5.2 and Proposition 5.5 respectively.

According to (2.1), we need to understand the space of pairs of $k$-linear maps

$$
\left\langle v_{1}, v_{2}\right\rangle_{k} \underset{A_{y}}{\stackrel{A_{x}}{\longrightarrow}} \mathfrak{m} \cdot M \text { satisfying } x \cdot\left(A_{y} v_{i}\right)=y \cdot\left(A_{x} v_{i}\right) \text { for } i=1,2 \text {. }
$$


Here $A_{x}$ and $A_{y}$ are two by two matrices corresponding to multiplication by $x$ and $y$ restricted to the $A$-linear generators $v_{1}$ and $v_{2}$. We need to consider the above data up to the equivalence relation that identifies pairs of matrices that give rise to isomorphic modules. This equivalence relation is determined in Lemma 5.4 below in the case where $\mathfrak{m} \cdot M=k \oplus k$.

Case (a) above is completely solved by the following result.

Proposition 5.2. The decomposable modules with $r=2$ and such that $\mathfrak{m} \cdot M$ is a structure sheaf of length 2 form an $\mathbb{A}^{1}$-fibration over $\mathbb{P}^{1}$, hence with motivic class $\mathbb{L}(\mathbb{L}+1)$. The indecomposable ones form a family $\mathcal{F}_{1}$ isomorphic to $\mathbb{P}^{1}$.

PROOF. If $M$ is generated as a $k$-vector space by $\left\{v_{1}, v_{2}, v_{3}, v_{4}\right\}$, we can assume the $k$-linear generators of $\mathfrak{m} \cdot M=A /\left(x^{2}, y-t x\right)$ to be $v_{3}=1$ and $v_{4}=x$. Here we have fixed $t \in \mathbb{A}^{1}=\mathbb{P}^{1} \backslash\{\infty\}$; then, after imposing the relations $x \cdot\left(y \cdot v_{i}\right)=y \cdot\left(x \cdot v_{i}\right)$ for $i=1,2$, the multiplication table for $M$ is

\begin{tabular}{ccccc} 
& $v_{1}$ & $v_{2}$ & $v_{3}$ & $v_{4}$ \\
\hline$x \cdot$ & $a_{1} v_{3}+b_{1} v_{4}$ & $a_{2} v_{3}+b_{2} v_{4}$ & $v_{4}$ & 0 \\
$y \cdot$ & $a_{1} t v_{3}+c_{1} v_{4}$ & $a_{2} t v_{3}+c_{2} v_{4}$ & $t v_{4}$ & 0 \\
\hline
\end{tabular}

and since $M$ is generated as an $A$-module by $v_{1}$ and $v_{2}$, we can assume $a_{1}=1$. Then, we may assume $a_{2}=0$ by replacing $v_{2}$ with $=v_{2}-a_{2} v_{1}$. Since $x \cdot v_{3}=v_{4}$, we can assume $b_{1}=b_{2}=0$ by replacing $v_{1}$ and $v_{2}$ with $v_{1}-b_{1} v_{3}$ and $v_{2}-b_{2} v_{3}$ respectively. This yields the multiplication table

\begin{tabular}{ccccc} 
& $v_{1}$ & $v_{2}$ & $v_{3}$ & $v_{4}$ \\
\hline$x \cdot$ & $v_{3}$ & 0 & $v_{4}$ & 0 \\
$y \cdot$ & $t v_{3}+z v_{4}$ & $c_{2}^{\prime} v_{4}$ & $t v_{4}$ & 0 \\
\hline
\end{tabular}

where $z$ and $c_{2}^{\prime}$ arise from the above changes of basis. Here we distinguish between two cases: either $c_{2}^{\prime}=0$ or $c_{2}^{\prime} \neq 0$. In the former case we obtain, for each $t \in \mathbb{A}^{1}$, a family of decomposable modules parametrized by $z \in \mathbb{A}^{1}$. This family extends to the whole $\mathbb{P}^{1}$ of double points, so that the full family is an $\mathbb{A}^{1}$-fibration over $\mathbb{P}^{1}$.

On the other hand, if $c_{2}^{\prime} \neq 0$, we may assume $c_{2}^{\prime}=1$. Replacing $v_{1}$ by $v_{1}-z v_{2}$, we may also assume $z=0$, so for each $t \in \mathbb{A}^{1}$ we have exactly one indecomposable module: its multiplication table is

\begin{tabular}{ccccc} 
& $v_{1}$ & $v_{2}$ & $v_{3}$ & $v_{4}$ \\
\hline$x \cdot$ & $v_{3}$ & 0 & $v_{4}$ & 0 \\
$y \cdot$ & $t v_{3}$ & $v_{4}$ & $t v_{4}$ & 0 \\
\hline
\end{tabular}

and this family again extends to $t=\infty$ giving a family $\mathcal{F}_{1} \cong \mathbb{P}^{1}$.

Remark 5.3. The decomposable modules of Proposition 5.2 are those of the form $k \oplus \mathscr{O}_{Z}$, where $Z \subset \mathbb{A}^{2}$ is a curvilinear subscheme of length 3. The motive $\mathbb{L}(\mathbb{L}+1)$ is indeed the class of the curvilinear locus inside $\operatorname{Hilb}^{3}\left(\mathbb{A}^{2}\right)_{0}$. The family $\mathcal{F}_{1}$ parametrizes the $k$-linear duals $(A / I)^{*}$, where $I \subset k[x, y]$ is a non-complete-intersection ideal. This family of subschemes of the plane, parametrized by the $\mathbb{P}^{1}$ of linear forms on $k^{2}$, was studied by Briançon [Bri77].

It remains to treat case (b), so from now on we assume our modules $M$ satisfy

$$
\mathfrak{m} \cdot M=k \oplus k \text {. }
$$



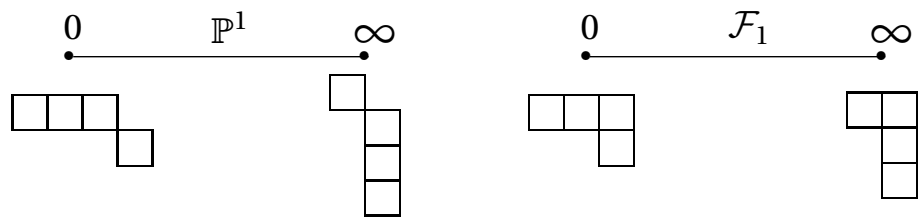

FIGURE 2. The left picture represents the $z=0$ slice of the family of decomposable modules of Proposition 5.2. The right picture is the family of indecomposable modules given by (5.1).

This assumption makes the relations $x \cdot\left(y \cdot v_{i}\right)=y \cdot\left(x \cdot v_{i}\right)$ vacuous. We thus want to describe the quasi-affine variety

$$
U=\left\{k \text {-linear maps } A_{x}, A_{y}:\left\langle v_{1}, v_{2}\right\rangle_{k} \rightrightarrows k \oplus k \mid \operatorname{rk}\left(A_{x} A_{y}\right)=2\right\}
$$

up to a suitable group action. Here $\left(A_{x} A_{y}\right)$ is the $2 \times 4$ matrix obtained by juxtaposing the two given square matrices. The rank condition comes from the requirement $r=$ 2. The next result characterizes pairs of matrices producing isomorphic modules.

LEMMA 5.4. Two pairs of matrices $\left(A_{x}, A_{y}\right)$ and $\left(B_{x}, B_{y}\right)$ as above give rise to isomorphic A-modules if and only if

$$
H A_{x} K=B_{x}, \quad H A_{y} K=B_{y}
$$

for some $H, K \in \mathrm{GL}_{2}$.

PROOF. An isomorphism of modules can be identified with a matrix in $\mathrm{GL}_{4}$, which we write in block form as

$$
\left(\begin{array}{cc}
W & X \\
Y & Z
\end{array}\right)
$$

The matrix $X$ describes a mapping $\left\langle v_{3}, v_{4}\right\rangle_{k} \rightarrow\left\langle v_{1}, v_{2}\right\rangle_{k}$. Since the submodule generated by $v_{3}$ and $v_{4}$ is $k^{2}$, then $x \cdot v_{3}=y \cdot v_{3}=x \cdot v_{4}=y \cdot v_{4}=0$. Then, the matrix $X$ contributes trivially to the isomorphism, so we can assume $X$ is the zero matrix. On the other hand, the matrix $Y$ describes a component mapping $\left\langle v_{1}, v_{2}\right\rangle_{k} \rightarrow\left\langle v_{3}, v_{4}\right\rangle_{k}$. Since we are assuming $v_{1}$ and $v_{2}$ to be generators, $Y$ must be the zero matrix. It follows than $W$ and $Z$ belong to $\mathrm{GL}_{2}$. A direct computation shows that $K=W$ and $H=Z^{-1}$.

In what follows, we study the quotient stack

$$
U / \mathrm{GL}_{2} \times \mathrm{GL}_{2}
$$

which gives a presentation of the substack of $\mathcal{X}_{2}(4)$ representing modules of type (b).

Proposition 5.5. The indecomposable modules of length 4 with $\mathfrak{m} \cdot M=k \oplus k$ form a family $\mathcal{F}_{2}$ isomorphic to $\mathbb{P}^{1}$.

Proof. The condition $\operatorname{rk}\left(A_{x} A_{y}\right)=2$ from (5.2) says that either

(1) $\operatorname{rk} A_{x}=\operatorname{rk} A_{y}=1$, or

(2) either $A_{x}$ or $A_{y}$ is invertible.

In the first case, up to the action of $\mathrm{GL}_{2} \times \mathrm{GL}_{2}$ we can assume

$$
A_{x}=\left(\begin{array}{ll}
1 & 0 \\
0 & 0
\end{array}\right) \text {. }
$$


At this point the multiplication table of a module with $\mathfrak{m} \cdot M=k \oplus k$ looks like

\begin{tabular}{ccccc} 
& $v_{1}$ & $v_{2}$ & $v_{3}$ & $v_{4}$ \\
\hline$x \cdot$ & $v_{3}$ & 0 & 0 & 0 \\
$y \cdot$ & $\alpha v_{3}+\beta v_{4}$ & $\gamma v_{3}+\delta v_{4}$ & 0 & 0 \\
\hline
\end{tabular}

and the conditions $\operatorname{rk} A_{x}=\operatorname{rk} A_{y}=1$ and $\operatorname{rk}\left(A_{x} A_{y}\right)=2$ ensure that $\alpha \delta=\beta \gamma$ and $(\beta, \delta) \neq(0,0)$, respectively. Assume $\delta \neq 0$. Then by replacing $v_{4}$ with $\gamma v_{3}+\delta v_{4}$ we can assume $\gamma=0, \delta=1$ and therefore $\alpha=0$. Further replacing $v_{1}$ by $v_{1}-\beta b_{2}$ yields the table

\begin{tabular}{ccccc} 
& $v_{1}$ & $v_{2}$ & $v_{3}$ & $v_{4}$ \\
\hline$x \cdot$ & $v_{3}$ & 0 & 0 & 0 \\
$y \cdot$ & 0 & $v_{4}$ & 0 & 0 \\
\hline
\end{tabular}

which is a single module. Similarly, if $\beta \neq 0$, replacing $v_{4}$ by $\alpha v_{3}+\beta v_{4}$ we can assume $\alpha=0, \beta=1$ and therefore $\gamma=0$. Further replacing $v_{2}$ by $v_{2}-\delta b_{1}$ we get

\begin{tabular}{ccccc} 
& $v_{1}$ & $v_{2}$ & $v_{3}$ & $v_{4}$ \\
\hline$x \cdot$ & $v_{3}$ & 0 & 0 & 0 \\
$y \cdot$ & $v_{4}$ & 0 & 0 & 0 \\
\hline
\end{tabular}

showing that case (1) contributes only two isomorphism classes of modules, both decomposable and representable by skew Ferrers diagrams (see Figure 3).

We are left to deal with the loci in (5.2) where $A_{x}$ is invertible and the one where $A_{y}$ is invertible. These are isomorphic along their common intersection. If, say, $A_{x}$ is invertible, by the action of $\mathrm{GL}_{2} \times \mathrm{GL}_{2}$ described in Lemma 5.4, we may assume $A_{x}$ is the identity matrix and $A_{y}$ is in Jordan form. If $A_{y}$ is not diagonalizable and has eigenvalue $\eta \in \mathbb{A}^{1}$, we get

$$
A_{x}=\left(\begin{array}{ll}
1 & 0 \\
0 & 1
\end{array}\right), \quad A_{y}=\left(\begin{array}{ll}
\eta & 1 \\
0 & \eta
\end{array}\right) .
$$

Joining this family with the module represented by the pair

$$
A_{x}=\left(\begin{array}{ll}
0 & 1 \\
0 & 0
\end{array}\right), \quad A_{y}=\left(\begin{array}{ll}
1 & 0 \\
0 & 1
\end{array}\right)
$$

gives a family of indecomposable modules $\mathcal{F}_{2}$ parametrized by $\mathbb{P}^{1}$. This exhausts the non diagonalizable case, and all other modules are decomposable: for instance, if $A_{x}$ is invertible and $A_{y}$ is diagonalizable with eigenvalues $(\lambda, \mu)$, we obtain the module

\begin{tabular}{ccccc} 
& $v_{1}$ & $v_{2}$ & $v_{3}$ & $v_{4}$ \\
\hline$x \cdot$ & $v_{3}$ & $v_{4}$ & 0 & 0 \\
$y \cdot$ & $\lambda v_{3}$ & $\mu v_{4}$ & 0 & 0 \\
\hline
\end{tabular}

which is the direct sum of two structure sheaves of length 2 .

Theorem 1 follows combining Proposition 5.2 and Proposition 5.5 with one another.

For completeness, let us finish the classification of decomposable modules. By the proof of the previous proposition, we are left to consider the locus, in $U$, where $A_{x}$ is invertible and $A_{y}$ is diagonalizable; this glues to the locus where $A_{y}$ is invertible and $A_{x}$ is diagonalizable. Up to the action of $\mathrm{GL}_{2} \times \mathrm{GL}_{2}$, when one between $A_{x}$ and $A_{y}$ is invertible, we can always assume the other to be in Jordan normal form. 

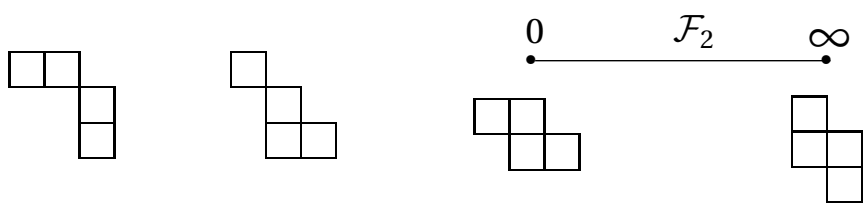

FIGURE 3. The left picture represents the two isolated modules (5.3) and (5.4). The right picture describes the family $\mathcal{F}_{2}=\mathbb{P}^{1}$ of indecomposable modules found in Proposition 5.5.

Joining the locus where $A_{x}$ is invertible and $A_{y}$ has two equal eigenvalues with the module represented by the pair

$$
A_{x}=\left(\begin{array}{ll}
0 & 0 \\
0 & 0
\end{array}\right), \quad A_{y}=\left(\begin{array}{ll}
1 & 0 \\
0 & 1
\end{array}\right)
$$

gives a family of decomposable modules

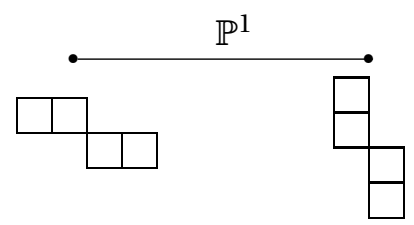

representing all length 4 modules of the form $\mathscr{O}_{Z} \oplus \mathscr{O}_{Z}$, where $Z \subset \mathbb{A}^{2}$ is a subscheme of length 2 . These have automorphism group

$$
\mathbb{G}_{a}^{4} \rtimes \mathrm{GL}_{2},
$$

which distinguishes them from the decomposables of the form $\mathscr{O}_{Z} \oplus \mathscr{O}_{Z^{\prime}}$ with $Z \neq Z^{\prime}$ two subschemes of length 2 . The latter indeed have automorphism group

$$
\mathbb{G}_{a}^{4} \rtimes \mathbb{G}_{m}^{2} .
$$

We have already encountered a module of this type, namely

$$
A /\left(y, x^{2}\right) \oplus A /\left(x, y^{2}\right),
$$

in the leftmost diagram of Figure 3. The other sums of (different) structure sheaves of length 2 arise by considering the remaining types of pairs $\left(A_{x}, A_{y}\right)$ up to $\mathrm{GL}_{2} \times \mathrm{GL}_{2}$. More precisely, we have the locus where $A_{x}$ is invertible and $A_{y}$ has distinct eigenvalues $\lambda \neq \mu$, and finally the $\mathbb{G}_{m}$ of modules represented by matrices

$$
A_{x}=\left(\begin{array}{ll}
0 & 0 \\
0 & v
\end{array}\right), \quad A_{y}=\left(\begin{array}{ll}
1 & 0 \\
0 & 1
\end{array}\right), \quad v \neq 0 .
$$

We now need to compute the motivic contribution of this family.

LEMMA 5.6. The motivic contribution of the modules $\mathscr{O}_{Z} \oplus \mathscr{O}_{Z^{\prime}}$, composed by the direct sum of two distinct structure sheaves of length 2 is

$$
\frac{\mathbb{L}\left(\mathbb{L}^{2}+1\right)}{\mathbb{L}+1} \text {. }
$$

Proof. It is clear that (5.5) and (5.6) together contribute $\mathbb{L}$. The remaining locus parametrizes Jordan forms of matrices with two distinct eigenvalues, namely

$$
\left\{(\lambda, \mu) \in \mathbb{A}^{1} \times \mathbb{A}^{1} \mid \lambda \neq \mu\right\} / \mathbb{Z}_{2} .
$$


We compute the motivic class $\xi$ of this locus formally, decomposing the motive of $\operatorname{End}\left(k^{2}\right)$ according to the Jordan type. We obtain the identity

$$
\mathbb{L}^{4}=\xi \cdot \frac{\left[\mathrm{GL}_{2}\right]}{(\mathbb{L}-1)^{2}}+\mathbb{L} \cdot \frac{\left[\mathrm{GL}_{2}\right]}{\mathrm{GL}_{2}}+\mathbb{L} \frac{\left[\mathrm{GL}_{2}\right]}{\mathbb{L}(\mathbb{L}-1)}
$$

where each "fraction" describes the orbit of a given Jordan form. The middle $\mathbb{L}$ parametrizes matrices of the form $\lambda \cdot I d$, and similarly the last $\mathbb{L}$ corresponds to non-diagonalizable Jordan forms. After solving for $\xi$, the total contribution is

$$
\xi+\mathbb{L}=\frac{\mathbb{L}^{3}-\mathbb{L}^{2}}{\mathbb{L}+1}+\mathbb{L}=\frac{\mathbb{L}\left(\mathbb{L}^{2}+1\right)}{\mathbb{L}+1},
$$

proving the lemma.

We summarize in Table 2 all families of modules of length 4 . The automorphism groups of all modules are computed through Theorem 3.3.

\begin{tabular}{cccc}
$r$ & $\mathcal{C}(4)_{0}$ & {$\left[\operatorname{Aut}_{A}(M)\right]$} & Motivic contribution \\
\hline 1 & $\mathscr{O}_{Z}$ & $\mathbb{L}^{3}(\mathbb{L}-1)$ & $\mathbb{L}^{3}+2 \mathbb{L}^{2}+\mathbb{L}+1$ \\
2 & $\mathcal{F}_{1}$ & $\mathbb{L}^{3}(\mathbb{L}-1)$ & $\mathbb{L}+1$ \\
2 & $\mathcal{F}_{2}$ & $\mathbb{L}^{5}(\mathbb{L}-1)$ & $\mathbb{L}+1$ \\
2 & $k \oplus A / \mathfrak{m}^{2}$ & $\mathbb{L}^{5}(\mathbb{L}-1)^{2}$ & 1 \\
2 & $k \oplus \mathscr{O}_{Z}, Z$ curvilinear & $\mathbb{L}^{4}(\mathbb{L}-1)^{2}$ & $\mathbb{L}(\mathbb{L}+1)$ \\
2 & $\mathscr{O}_{Z} \oplus \mathscr{O}_{Z}$ & $\mathbb{L}^{4}\left[\mathrm{GL} L_{2}\right]$ & $\mathbb{L}+1$ \\
2 & $\mathscr{O}_{Z} \oplus \mathscr{O}_{Z^{\prime}}, Z \neq Z^{\prime}$ & $\mathbb{L}^{4}(\mathbb{L}-1)^{2}$ & $\frac{\mathbb{L}\left(\mathbb{L}^{2}+1\right)}{\mathbb{L}+1}$ \\
3 & $k^{2} \oplus \mathscr{O}_{Z}$ & $\mathbb{L}^{5}(\mathbb{L}-1)\left[\mathrm{GL}_{2}\right]$ & $\mathbb{L}+1$ \\
3 & $k \oplus\left(A / \mathfrak{m}^{2}\right)^{*}$ & $\mathbb{L}^{5}(\mathbb{L}-1)^{2}$ & 1 \\
4 & $k^{4}$ & {$\left[\mathrm{GL}_{4}\right]$} & 1 \\
\hline
\end{tabular}

TABLE 2. All length 4 modules supported at the origin, along with the class of their automorphism group and the corresponding motivic contribution. The first three rows describe indecomposable modules.

Remark 5.7. The coefficient of $t^{4}$ in (3.1) can be computed to be

$$
\left[\mathcal{C}(4)_{0}\right]=\frac{1}{\left[\mathrm{GL}_{4}\right]}\left(\mathbb{L}^{15}+2 \mathbb{L}^{14}+\mathbb{L}^{13}+\mathbb{L}^{12}-2 \mathbb{L}^{11}-2 \mathbb{L}^{10}-\mathbb{L}^{9}+\mathbb{L}^{7}\right) .
$$

The sum of the motives in the rightmost column of Table 2, each divided by the motive of the corresponding automorphism group, recovers precisely this class, confirming our calculation. 


\section{TORUS ACTION}

Let $V$ be an $n$-dimensional vector space, and consider the commuting variety

$$
C_{n}=\left\{(X, Y) \in \operatorname{End}(V)^{2} \mid[X, Y]=0\right\} \subset \operatorname{End}(V)^{2} .
$$

It contains the closed subscheme $N_{n}$ of pairs of commuting nilpotent matrices. The group $\mathrm{GL}_{n}$ acts on these spaces by simultaneous conjugation, and the closed immersion of quotient stacks

is equivalent to the closed immersion

$$
N_{n} / \mathrm{GL}_{n} \subset C_{n} / \mathrm{GL}_{n}
$$

$$
\mathcal{C}(n)_{0} \subset \mathcal{C}(n)
$$

of the stack of coherent sheaves supported at the origin inside the full stack of coherent sheaves of length $n$. The natural action of the torus $\mathbf{T}=\mathbb{G}_{m}^{2}$ on $\mathbb{A}^{2}$, given by rescaling coordinates,

$$
\left(t_{1}, t_{2}\right) \cdot(x, y)=\left(t_{1} x, t_{2} y\right)
$$

can be lifted to $\mathrm{GL}_{n}$-equivariant actions on $C_{n}$ and $N_{n}$. This gives an induced Taction on $\mathcal{C}(n)$, leaving $\mathcal{C}(n)_{0}$ invariant. We show in Proposition 6.2 below that this torus action has finitely many fixed points. This will finally make precise the connection with skew Ferrers diagrams, which we used as a mere graphical representation so far.

Recall that a skew Ferrers diagram is a difference of two Ferrers diagram; a particularly interesting class of skew Ferrers diagrams are parallelogram polyominoes, studied for instance in [Pól69, DF93].

Definition 6.1. A parallelogram polyomino is a skew Ferrers diagram that is connected and has no cut point. We illustrate the terminology in Figure 4 below.
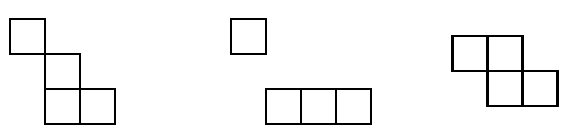

FIGURE 4. From left to right: a connected skew Ferrers diagram with a cut point, a disconnected skew Ferrers diagram, a parallelogram polyomino of area 4 .

Proposition 6.2. The $\mathbf{T}$-fixed locus $\mathcal{C}(n)^{\mathbf{T}} \subset \mathcal{C}(n)$ lies in $\mathcal{C}(n)_{0}$ and is finite. The indecomposable $\mathbf{T}$-fixed modules are in bijection with the set of parallelogram polyominoes.

Proof. The support of a torus-fixed module is a torus-fixed subscheme of $\mathbb{A}^{2}$, so $\mathcal{C}(n)^{\mathbf{T}} \subset \mathcal{C}(n)_{0}$. Let $F \in \mathcal{C}(n)^{\mathbf{T}}$ be a torus fixed $A$-module. Then $F$ corresponds to a T-representation

$$
\rho: \mathbf{T} \rightarrow \mathrm{GL}(V)
$$

of the underlying vector space $V$ such that the maps $\rho_{t}: V \stackrel{\rightarrow}{\rightarrow} V$ are $A$-linear isomorphisms. Let $\Gamma=\mathbb{Z}^{2}$ be the character lattice of the torus. There is a $k$-linear decomposition

$$
V=\bigoplus_{\chi \in \Gamma} V_{\chi}
$$

into irreducible subrepresentations

$$
V_{\chi}=\left\{v \in V \mid \rho_{t}(v)=\chi(t) v \text { for all } t \in \mathbf{T}\right\} .
$$


So $V$ has an eigenbasis $\mathbf{v}=\left\{v_{1}, \ldots, v_{n}\right\}$ indexed by characters $\chi_{1}, \ldots, \chi_{n} \in \Gamma$. By $A$ linearity of $\rho_{t}$, for every $i$ we have the relation

$$
\rho_{t}\left(x \cdot v_{i}\right)=x \cdot \rho_{t}\left(v_{i}\right)=\chi_{i}(t)\left(x \cdot v_{i}\right) .
$$

In other words, $x \cdot v_{i}$ is either 0 or belongs to $\mathbf{v}$. The same holds for $y \cdot v_{i}$ by the same reasoning. We have shown that a torus fixed module has a $k$-linear basis $\mathbf{v}$ such that $x \cdot v_{i}$ and $y \cdot v_{i}$ both lie in $\mathbf{v} \cup\{0\}$. The set

$$
\left\{\chi_{1}, \ldots, \chi_{n}\right\} \subset \Gamma
$$

determines a skew Ferrers diagram of area $n$, hence $\mathcal{C}(n)^{\mathrm{T}}$ is finite. Finally, any cut point of a skew Ferrers diagram determines two proper submodules of the corresponding module, and these are necessarily direct summands. Conversely, a decomposable (torus-fixed) module can be represented by joining several parallelogram polyominoes creating cut points.

Consider the numbers

$$
c_{n}=\mid\left\{F \in \mathcal{C}(n)^{\mathbf{T}} \mid F \text { indecomposable }\right\} \mid
$$

and their generating function

$$
\sum_{n=0}^{\infty} c_{n} q^{n}=1+q+2 q^{2}+4 q^{3}+9 q^{4}+20 q^{5}+\cdots
$$

The main result of [DF93] is the calculation of the generating function $F$ of the numbers of parallelogram polyominoes with prescribed area and number of columns. The result is

$$
F(t ; q)=\sum_{n=0}^{\infty} \frac{(-1)^{n} q^{\left(\begin{array}{c}
n+1 \\
2
\end{array}\right)}}{(q ; q)_{n}(q ; q)_{n+1}} q^{n+1} t^{n+1} / \sum_{n=0}^{\infty} \frac{(-1)^{n} q^{\left(\begin{array}{c}
n \\
2
\end{array}\right)}}{(q ; q)_{n}^{2}} q^{n} t^{n},
$$

where $(a ; q)_{n}=\prod_{i=0}^{n-1}\left(1-a q^{i}\right)$ is the $q$-Pochhammer symbol and $q$ (resp. $\left.t\right)$ is the variable that keeps track of the area (resp. number of columns). It follows from Proposition 6.2 that one can compute

$$
\sum_{n=0}^{\infty} c_{n} q^{n}=F(1 ; q)
$$

Acknowledgements. The first named author is supported by the Department of Mathematics and Natural Sciences of the University of Stavanger in the framework of the grant 230986 of the Research Council of Norway. We wish to thank Martin G. Gulbrandsen for the many insightful conversations around the topics discussed here. We also thank Jim Bryan, Aurelio Carlucci, Roy Skjelnes and Mattia Talpo for their valuable comments during the preparation of this work. 


\section{REFERENCES}

[BBS13] Kai Behrend, Jim Bryan, and Balázs Szendrői, Motivic degree zero Donaldson-Thomas invariants, Invent. Math. 192 (2013), no. 1, 111-160.

[BM15] Jim Bryan and Andrew Morrison, Motivic classes of commuting varieties via power structures, J. Algebraic Geom. 24 (2015), no. 1, 183-199.

[Bri77] Joel Briançon, Description de Hilb ${ }^{n} \mathbb{C}\{x, y\}$, Invent. Math. 41 (1977), 45-89.

[Bri12] Michel Brion, Representations of quivers, Geometric methods in representation theory I, Sémin. Congr., vol. 24, Soc. Math. France, Paris, 2012, pp. 103-144.

[DF93] M. P. Delest and J. M. Fedou, Enumeration of skew ferrers diagrams, Discrete Mathematics 112 (1993), no. 1, 65-79.

[Eke09] Torsten Ekedahl, The Grothendieck group of algebraic stacks, arXiv : 0903.3143v2, 2009.

[ES87] Geir Ellingsrud and Stein Arild Strømme, On the homology of the Hilbert scheme of points in the plane, Invent. Math. 87 (1987), 343-352.

[FF60] Walter Feit and Nathan J. Fine, Pairs of commuting matrices over a finite field, Duke Math. J 27 (1960), 91-94.

[GLM04] S.M. Gusein-Zade, I. Luengo, and A. Melle-Hernández, A power structure over the Grothendieck ring of varieties, Math. Res. Lett. 11 (2004), no. 1, 49-57.

[Göt01] Lothar Göttsche, On the motive of the Hilbert scheme of points on a surface, Math. Res. Lett. 8 (2001), 613-627.

[GP69] I. M. Gelfand and V.A. Ponomarev, Remarks on the classification of a pair of commuting linear transformations in a finite dimensional vector space, Funct. Anal. 3 (1969), 325-326.

[Pól69] G. Pólya, On the number of certain lattice polygons, J. Combinatorial Theory 6 (1969), 102105.

[Poo08] Bjorn Poonen, Isomorphism types of commutative algebras of finite rank over an algebraically closed field, Computational arithmetic geometry, Contemp. Math., vol. 463, Amer. Math. Soc., Providence, RI, 2008, pp. 111-120.

[Ste11] Ernst Steinitz, Rechteckige Systeme und Moduln in algebraischen Zahlköppern. I, Mathematische Annalen 71 (1911), no. 3, 328-354. 